\title{
Numerical Simulation of Incompletely Premixed Oblique Detonation Stabilized on a Solid Surface
}

\author{
By Kazuya Iwata, ${ }^{1)}$ Shinji NAKAYA ${ }^{1)}$ and Mitsuhiro TsuE ${ }^{1)}$ \\ ${ }^{1)}$ Department of Aeronautics and Astronautics, The University of Tokyo, Tokyo, Japan
}

(Received July 31st, 2015)

\begin{abstract}
Oblique detonation under incompletely premixed conditions has not been well understood and is of great concern when difficulty of high-speed premixing in Oblique Detonation Wave Engine (ODWE), which is one of the most potential hypersonic aerospace propulsion systems, is taken into account. This study numerically investigated effects of fuel concentration gradients on oblique detonation and shock-induced combustion formed on a $28.20^{\circ}$ wedge by solving two-dimensional Navier-Stokes equations with a detailed chemical kinetic mechanism of hydrogen-air combustion. Oblique detonation with smooth-transition formed at a Mach number of 8.00, a static temperature of $300 \mathrm{~K}$, and a static pressure of $8.50 \mathrm{kPa}$ was referred as the completely premixed case. Fuel concentration gradients were described by the Gaussian function. At the maximum equivalence ratio of 2.00, Smooth-transition was replaced by abrupt-transition. When maximum equivalence ratio exceeded 3.00, a V-shaped flame front appeared with its leading edge located away from the wedge, which caused two separate triple-points to be observed. Second triple point appeared at the intersection of the incident shock or the detonation front and a reflected shock generated by compression waves on the lower side of the deflagration front. Increase of the front angle enabled intensive combustion to be maintained downstream of it.
\end{abstract}

Key Words: Oblique Detonation, Shock-Induced Combustion, ODWE, Incomplete Premixing, Wedge

\section{Nomenclature}

a : steepness of the gradients

$H \quad$ : position normal to the inflow [m]

$l \quad:$ induction length $[\mathrm{m}]$

$T \quad:$ temperature $[\mathrm{K}]$

$x \quad:$ longitudinal position on the wedge [m]

$X \quad:$ mole fraction [-]

$y \quad:$ position normal to the wedge [m]

$\theta \quad:$ half-wedge angle $\left[{ }^{\circ}\right]$

$\Phi \quad$ : equivalence ratio [-]

Subscripts

$0 \quad:$ centerline

\section{Introduction}

A chemically powered rocket for vertical launch has been our major earth-to-orbit transportation system with its increasing reliability and actual achievements. Today, a totally reusable spaceplane is attracting increasing attention for its potential of improving substantial problems of the conventional rockets, such as their payload capacities and launching costs. ${ }^{1,2)}$

An air-breathing engine, instead of the conventional rocket engines, is suited for such reusability due to its high specific thrust. ${ }^{1,2)}$ No oxidizer needs to be mounted, which enables us to take more payload weight to the orbit. In addition, since the spaceplane takes off and lands in the same way as conventional airplanes do, it will be more reliable and safe.

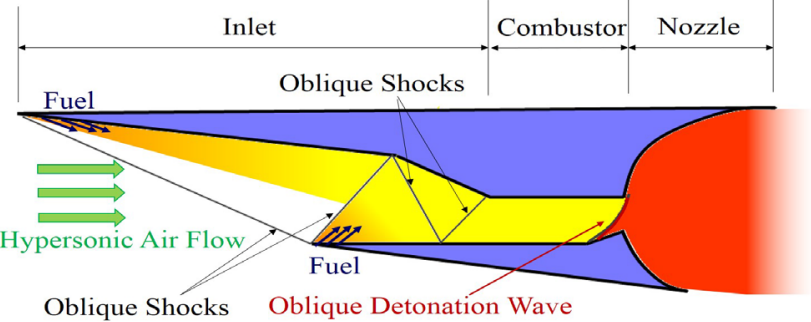

Fig. 1. Schematic of ODWE. ${ }^{1)}$

However, there are major problems to be tackled with a hypersonic air-breathing propulsion system in order to realize the spaceplane. Scramjet engine, ${ }^{3,4)}$ which is one of the most potential engines for a hypersonic flight, achieves diffusive combustion in a supersonic flow, inevitably accompanied by difficulty of mixing, flame stabilization and loss of potential thrust by shock waves. A lot of researchers have worked on those inherent problems from both experimental and numerical approaches, but they are yet to be solved and Scramjet has not come into a practical use.

Oblique Detonation Wave Engine (ODWE) is another concept which can potentially overcome the problems mentioned above. ${ }^{1,5-7)}$ ODWE accomplishes mixing upstream of the combustor in the inlet or on the engine forebody, by which supersonic premixed combustion (oblique detonation) is generated in the combustor (Fig. 1) instead of diffusive burning occurring in a Scramjet combustor. There is no difficulty of mixing and stabilization.

Detonation is defined as a strong interaction of shock fronts 
and flame fronts formed in a premixed combustible mixture. ${ }^{89}$ A hypersonic flow exceeding its flame speed stabilizes it on a solid surface as oblique detonation. It is completed in such a short distance that the combustor can be shortened, which can alleviate weight and cooling problems of hypersonic vehicles. ${ }^{5-7)}$ Furthermore, detonation intrinsically involves a compression process, which in turn decreases compression work needed to be done by the intake. ${ }^{5-7)}$

However, since premixing in ODWE is done in a hypersonic flow, it inevitably results in an inhomogeneous mixture. ${ }^{10)}$ Several researchers have been working on improvement of premixing in ODWE, ${ }^{11,12)}$ but we also have to consider effects of incomplete premixing on oblique detonation to evaluate possibility of flame stabilization and to resultant performance degradation of ODWE.

A lot of works have been done on fundamental physics of oblique detonation, ${ }^{13-16)}$ but almost all of them assume perfectly premixed conditions. Understanding of oblique detonation in a concentration gradient is lacking. A few researchers discussed effects of non-uniformity, ${ }^{10,17,18)}$ but their investigations were limited to a few parameters.

Objective of our research is then to understand effects of a concentration gradient on structures of oblique detonation and shock-induced combustion (defined as a decoupled shock and flame front) through a parametric numerical study, and to discuss physics involved in their features.

In the present study, an oblique detonation on a wedge observed in numerical works by Silva and Deshaies ${ }^{19)}$ was referred here as the completely premixed case. Concentration gradients were introduced into the incoming mixture prescribing them by the Gaussian function. ${ }^{10,17,18)}$

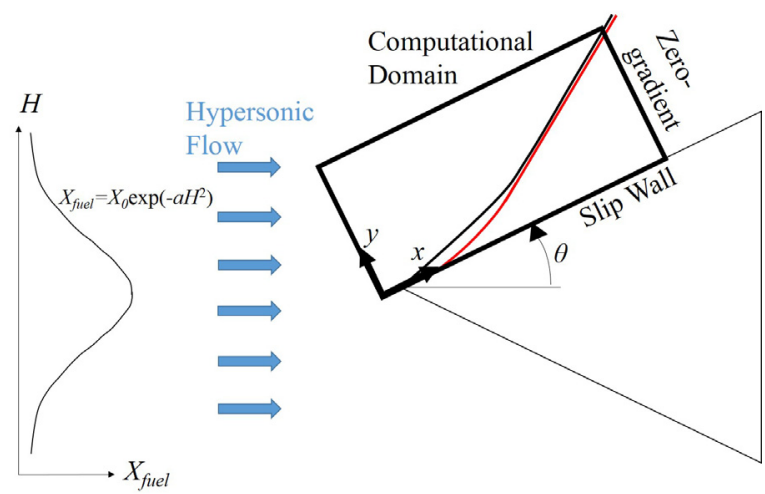

Fig. 2. The computational domain.

Table 1. Incoming hydrogen concentration gradients in the present study.

\begin{tabular}{|c|c|c|}
\hline Case & $\Phi_{\max }$ & $\Phi_{S}$ \\
\hline 1 & 1.50 & 0.44 \\
\hline 2 & 2.00 & 0.24 \\
\hline 3 & 2.50 & 0.15 \\
\hline 4 & 3.00 & 0.09 \\
\hline 5 & 4.00 & 0.05 \\
\hline 6 & 5.00 & 0.03 \\
\hline 7 & 6.00 & 0.02 \\
\hline 8 & 7.00 & 0.01 \\
\hline 9 & 8.00 & 0.01 \\
\hline
\end{tabular}

\section{Numerical Procedure}

\subsection{The computational domain}

As mentioned in the previous section, we performed a parametric numerical study on oblique detonation and shock induced combustion formed on a wedge in non-uniform mixtures. The present computational domain is in the shape of a rectangle, covering the surface of a wedge with the half angle of $\theta=28.20^{\circ}$, as shown in Fig. 2. The left and upper line of the domain are inlet boundaries where non-uniform mixtures come in at the angle of $\theta$. The right line corresponds to an outlet boundary on which zero-gradient condition of flow properties is assumed. The first $1.00 \mathrm{~mm}$ part of the lower line is also a zero-gradient outlet boundary, with the rest being a slip-wall boundary as the surface of the wedge. We performed preliminary simulations and verified that boundary layer had negligible influence on the structures obtained with minor differences of locations of the shock fronts and the flame fronts.

We employed two uniformly-spaced orthogonal grids, one of which is a standard grid $(801 \times 601$ for $10.00 \mathrm{~cm} \times 3.00$ $\mathrm{cm})$ and the other is an extended grid $(1001 \times 601$ for 12.50 $\mathrm{cm} \times 3.00 \mathrm{~cm})$. Resolution of the two grids is the same: $\Delta x$ $=125 \mu \mathrm{m}$, and $\Delta y=50 \mu \mathrm{m}$. This resolution allows about 10 grids to be included in half reaction length for the completely premixed condition.

\subsection{The governing equations}

Two-dimensional laminar Navier-Stokes equations were solved, including 9 species transport equations involved in a hydrogen-air combustion mechanism proposed by Konnov consisting of 27 elementary reactions. ${ }^{20)}$ Viscous terms were included here to survey effects of diffusive phenomena in strong concentration gradients.

Convection terms were discretized using Harten-Yee's 2nd-order upwind TVD scheme. ${ }^{21)}$ Diffusive terms were discretized with the second-order central difference.

Time integration was implemented fully implicitly, combining LU-SGS for overcoming CFL limitation and the diagonalized Point Implicit Method proposed by $\mathrm{Ju}^{22,23)}$. In this study, unsteadiness did not appear even when triple points emerged, due to the absence of unstable transverse waves. Therefore, only steady state solutions were addressed in our work.

\subsection{Concentration gradients}

Silva and Deshaies numerically observed a smooth front oblique detonation with smooth-transition, in which transition to detonation occurs with a smooth increase of the incident shock angle, under the condition where a uniformly stoichiometric hydrogen-air mixture flows onto a $28.20^{\circ}$ wedge at Mach 8 , a static temperature of $300 \mathrm{~K}$, and a static pressure of $8.50 \mathrm{kPa}^{19)} \mathrm{We}$ referred the above phenomenon as the completely premixed case.

Concentration gradients were introduced into the incoming mixture described above on the assumption that fuel volumetric fraction is distributed according to the Gaussian function as Eq. (1) shows, which is justified by measurements of hydrogen distribution injected into supersonic flows. ${ }^{24,25)}$ 


$$
X_{\text {fuel }}=X_{0} \exp \left(-a H^{2}\right)
$$

$H$ is vertical position normal to the inflow the origin of which is located on the tip of the wedge. $X_{0}$ and $a$ denote parameters describing the maximum mole fraction on the tip of the wedge and the steepness of a concentration gradient respectively. Their values were chosen so that total equivalence ratio is unity over the region where the completely premixed oblique detonation exists in the standard grid, corresponding to $0<H$ $<H_{S}$ ( $H_{S}$ is height of the intersection of the detonation front and the outlet boundary line). Integrated concentration in this range of height is used to calculate total equivalence ratio. Presently chosen nine concentration gradients are listed in Table 1 as the maximum equivalence ratios on the centerline $\Phi_{\max }$, and the resultant outermost values $\Phi_{S}$ at $H=H_{S}$. Velocity, a static pressure and a static temperature were all kept constant.

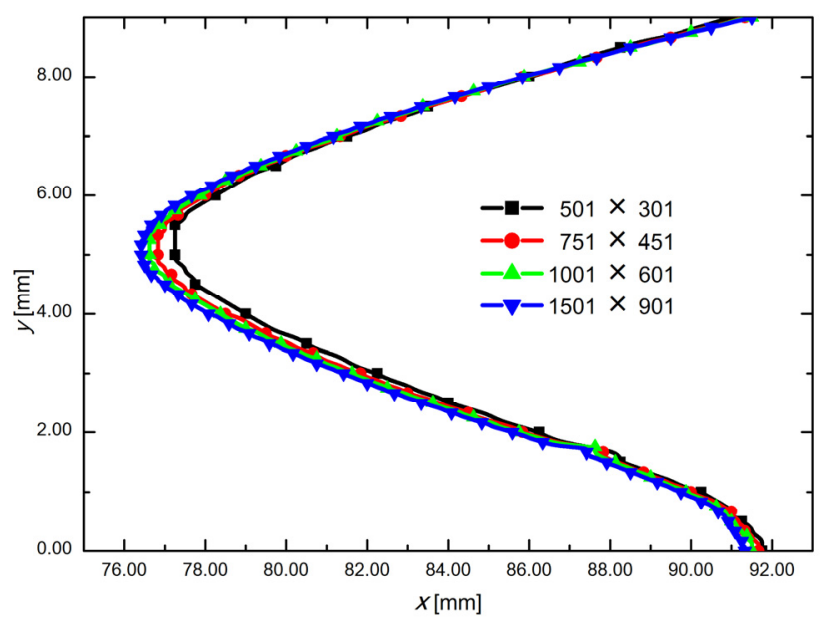

(a) The location of the V-shaped flame front tip

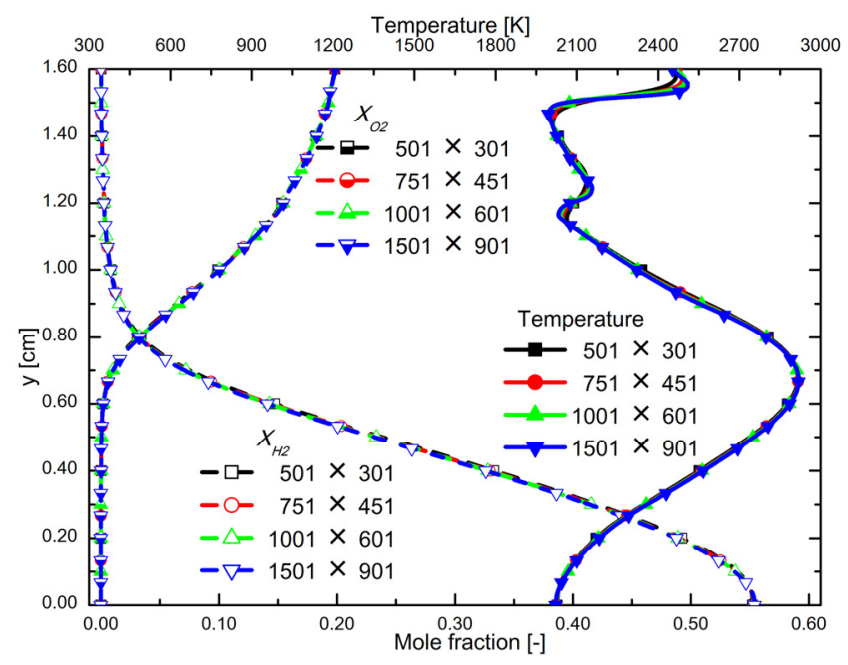

(b) Distribution of reactants and temperature at the outlet boundary

Fig. 3. Grid dependency of oblique detonation in Case 6.

\section{Results and Discussion}

\subsection{Grid dependency}

This study primarily aims to investigate general structures associated with the locations of shock fronts and flame fronts, and combustion behaviors of oblique detonation observed. Therefore, grid dependencies of locations of the fronts and thermal and chemical properties of combustion products at the outlet boundary were evaluated to establish that the presently employed grid is well resolved enough to make the grid convergence problem negligible.

Oblique detonation observed in Case $6\left(\Phi_{\max }=5.00\right)$ were used for grid dependency evaluation. As will be mentioned later, this condition exhibited a V-shaped flame front and transitions on two separate triple points, which are the most characteristic features in this study and thought to be strongly resolution sensitive. The extended grids were used with the numbers $501 \times 301,751 \times 451,1001 \times 601$ which is our present grid number, and $1501 \times 901$ as the most refined one.

Computations using the above grids revealed that the most part of the shock fronts and the flame fronts and combustion behaviors were insensitive to the resolution. Fig. 3(a) shows grid dependency of the location of the V-shaped flame front which was proved to be the most sensitive in the whole structure. Notable difference of the location around the apex can be seen between the $501 \times 301$ grid and the $751 \times 501$ one, but difference around the $1001 \times 601$ grid was small enough to assume a good grid convergence in our computations. Fig. 3(b) shows grid dependencies of temperature and mole fractions of reactants at the outlet boundary as the combustion behaviors. It is obvious that they are almost independent of the resolution. Outlet temperature shows a little dependency away from the surface, but the result of the present grid is coincident with the most refined one. The results obtained here in Fig. 3 justify using the $1001 \times 601$ grid.

\subsection{Completely premixed case}

The incompletely premixed conditions shown in Table 1 were simulated along with the uniform case for validation. The results by the standard grid $(801 \times 601$ for $10.00 \mathrm{~cm} \times$ $3.00 \mathrm{~cm}$ ) are displayed in Fig. 4, where pressure contours (gray) and water density contours (colored) were overlaid to clearly resolve the locations of shock fronts and flame fronts. Three cases among relatively mild non-uniform conditions (Case 1-4) are illustrated here. The lower line of each figure corresponds to the surface of the wedge. Premixed mixtures come in from the left upper side at the half wedge angle $\theta$. The left oblique line upstream of the incident shock is a streamline with the stoichiometric composition and the right one corresponds to the height of $H=H_{S}$, respectively.

The uniform case (Fig. 4(a)) exhibited smooth-transition, as Silva and Deshaies observed in the same condition. ${ }^{19)}$ It consists of (A) an incident shock front, (B) a deflagration front with its leading edge on the wedge, and (C) an oblique detonation front downstream of a transition point called 'triple point' as the intersection of (A) and (B). ${ }^{8,9)}$ However, in smooth-transition observed here, gradual increase of the shock angle makes the triple point unclear. 


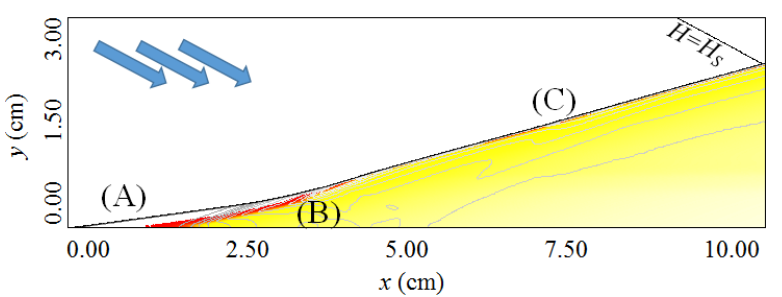

(a) Completely premixed case

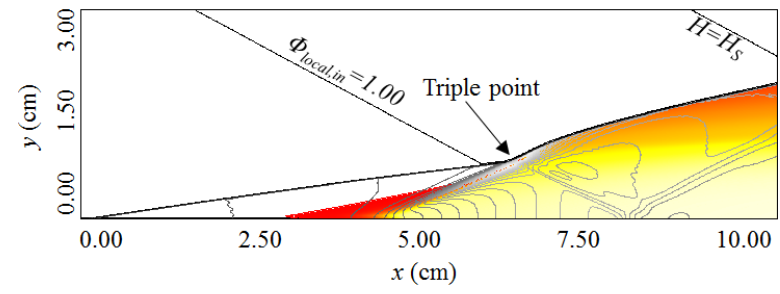

(c) Case 2, $\Phi_{\max }=2.00$

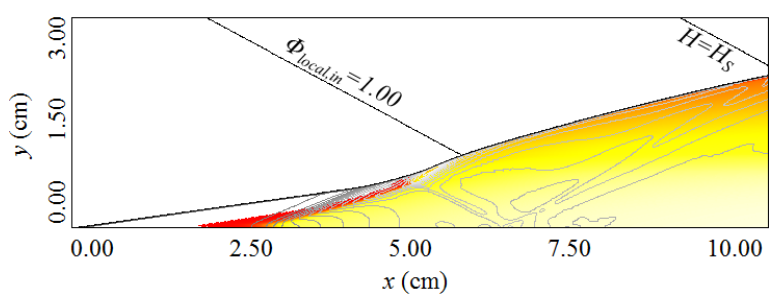

(b) Case 1, $\Phi_{\max }=1.50$

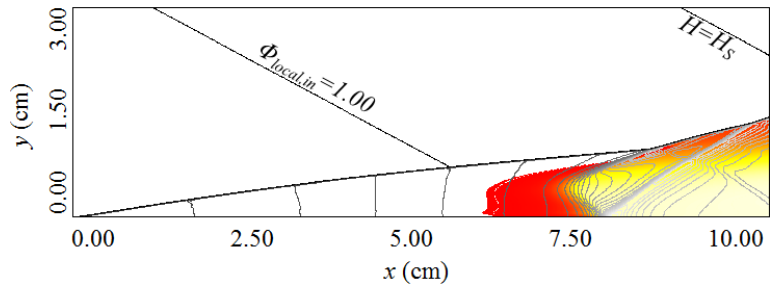

(d) Case 4, $\Phi_{\max }=3.00$

Fig. 4. Pressure (gray) and water density (colored) contours with $801 \times 601$ grid.

Deflagration on the surface begins more downstream than that in computation by Silva and Deshaies due to difference of chemical kinetic mechanisms employed. However, this difference did not affect the whole feature of oblique detonation, with the shock angle and the detonation angle $\left(35.00^{\circ}\right.$ and $44.00^{\circ}$ respectively in the result by Silva and Deshaies) reproduced well: the former angle was calculated to be $35.79^{\circ}$ in our computation, and the latter $44.00^{\circ}$. Distributions of temperature and pressure also agree well quantitatively (not shown in this paper). Therefore, it can be concluded that our computational method was well validated against oblique detonation phenomena we are concerned with.

\subsection{Incompletely premixed cases}

When concentration gradients were introduced, some fundamental changes to oblique detonation were observed. The weakest non-uniformity, Case 1 (Fig. 4(b)) caused the deflagration front to recede largely. Apparent triple point did not appear, but increase of the shock angle at the transition became evident and somewhat discontinuous. These changes became more remarkable in Case 2 (Fig. 4(c)) to see abrupt-transition with emergence of a distinct triple point. Transition at the triple point generated a peak pressure, and the highest flame temperature among all the cases in this study $(=3137.23 \mathrm{~K})$ occurred downstream of it. In every case here, high temperature around $3000 \mathrm{~K}$ was retained over the whole region in spite of non-uniform local equivalence ratio. Curved shock and detonation fronts observed here were caused by variable shock strength as the result of non-uniform composition generating distribution of incoming Mach number. ${ }^{17)}$ Flow behind the curved incident shock is parallel to the surface as it is in the uniform case.

Steeper gradients in Case 4 further pushed the flame front backward (Fig. 4(d)), which made it difficult to discuss their structures. The extended $1001 \times 601$ grid was then used to compute the more strongly non-uniform conditions (Case 4-9). The results of four cases among them which show characteristic features are displayed in Fig. 5 as overlaid contours of pressure (gray) and water density (colored).

One of the most characteristic features of the structures in Case 4-9 are found on deflagration front tips which are $\mathrm{V}$-shaped and projecting upstream, differently from those in the uniform case and milder non-uniformity cases (Case1-3, Fig .4(a)-(c)). This is due to delayed induction on the surface caused by highly rich concentration. In addition, since hydrogen-rich composition decreases incoming Mach number due to its lower molecular weight, post-shock temperature and pressure are also lower than those away from the surface. This peculiar shape can never be seen in uniformly premixed conditions where the leading edge of a deflagration front is always located on the surface. As shown in Fig. 5, the $\mathrm{V}$-shape becomes more acute as non-uniformity increases since the surface becomes more fuel-rich, which resulted in lower flame temperature on the wedge. However, location of the apex of the $\mathrm{V}$-shaped flame front did not change contrary to our expectation, for which overall detonation structures did not recede notably in contrast to weakly non-uniform conditions (Case1-3, Fig. 4). This will be discussed in detail later.

Another important feature is that triple points appear twice along the incident shock front or the detonation front. This can be seen clearer in Fig. 5(b) and (c). Second triple point apparently emerges as the intersection of the incident shock or the detonation front and a reflected shock initiating close to the foot of the $\mathrm{V}$-shaped flame front on the wedge. This reflected shock can be seen in pressure contours in Fig. 5 where discontinuous increase occurs. Plural triple points can be never seen in uniformly premixed oblique detonation except for unstable one with cellular structures. ${ }^{26)}$ Location of the first triple point did not change in Case 4-9, but that of second one went downstream as non-uniformity increased. Increase of the shock angle in Case 7 (Fig. 5(d)) is relatively gradual, which makes their locations unclear. 


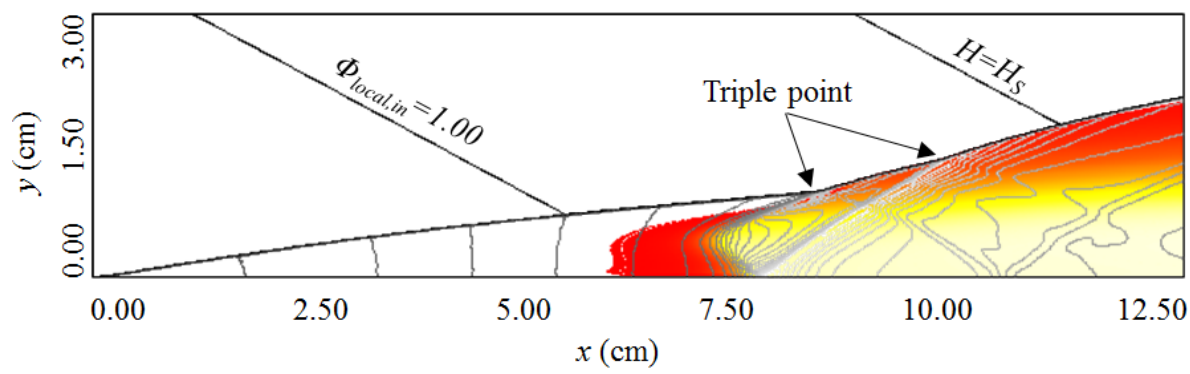

(a) Case $4, \Phi_{\max }=3.00$

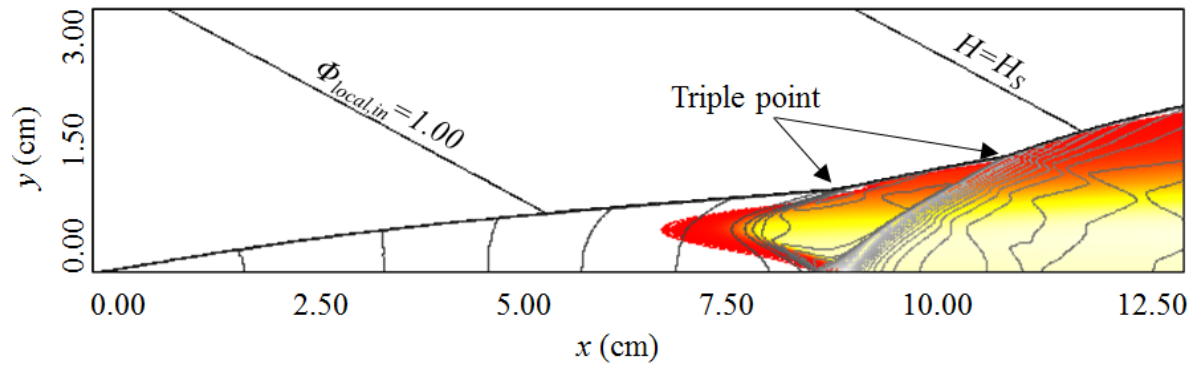

(b) Case 5, $\Phi_{\max }=4.00$

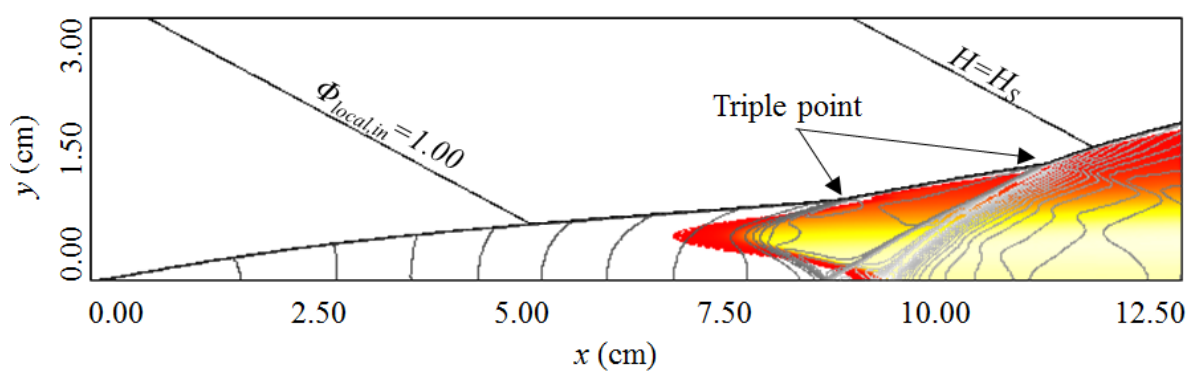

(c) Case 6, $\Phi_{\max }=5.00$

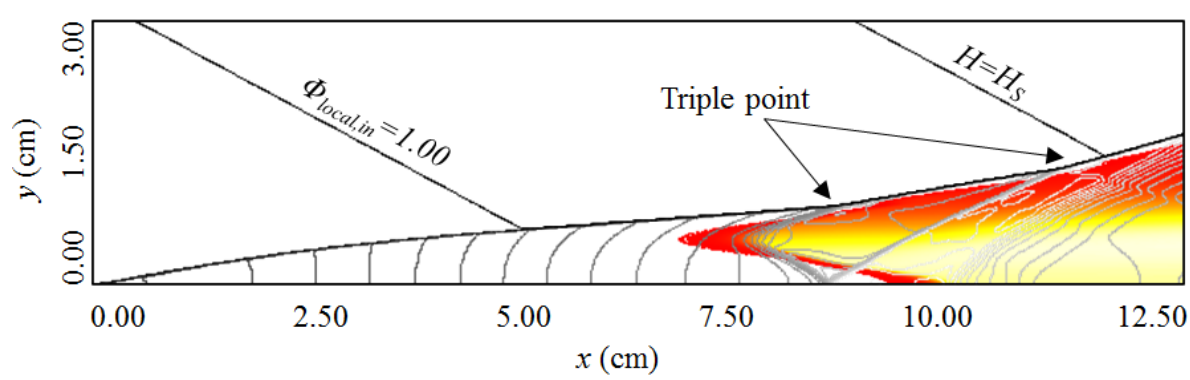

(d) Case 7, $\Phi_{\max }=6.00$

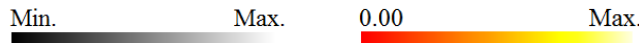

Fig. 5. Pressure (gray) and water density (colored) contours with $1001 \times 601$ grid.

The incident shock or the detonation front experiences increase in its angle twice on triple points, by which oblique detonation downstream of the first one in Case 4 (Fig. 5(a)) is intensified by the second one with a steeper angle downstream of it. In Case 5 and 6 , a more characteristic phenomenon occurred: Transition to detonation as well as decoupling of the shock and flame fronts occurred twice along the incident shock (Fig. 5(b), (c)). In Case 7 (Fig. 5(d)), on the contrary, transition to detonation no longer occurs on the triple points, resulting in shock-induced combustion over the whole region. However, increase of the shock angle still intensifies combustion with higher temperature downstream. Thus, plural triple points make it possible to retain intensive combustion away from the wedge even in a highly fuel-lean region.

\subsection{Second triple point}

Detailed physics of plural triple points can be explained by the mechanisms illustrated in Fig. 6: Fig. 6(a) shows a simplified structure of uniformly premixed oblique detonation, and Fig. 6(b) shows that of non-uniformly premixed oblique detonation with a V-shaped flame front. First triple point in non-uniform composition (Fig. 6(b)) occurs at the intersection of an incident shock and accumulating compression waves 
initiating on the upper side of the deflagration front, as it does under uniformly premixed conditions (Fig. 6(a)). These compression waves can be clearly seen in pressure contours near the apexes of the deflagration fronts in Fig. 5. On the other hand, second triple point is generated at the intersection of an incident shock front (or a detonation front) and a reflected shock initiating on the surface behind a deflagration front tip. This reflected shock depicted in Fig. 6(b) is the result of accumulation of compression waves starting on the lower side of the deflagration front. These compression waves are directed downward, accumulating into a single shock front on the surface. Overlaid contours in Fig. 5 show downgoing compression waves under the deflagration fronts and reflected shocks downstream of them. Therefore, second triple point can be regarded as the result of a V-shaped flame front, and that is why second triple point does not appear under weakly non-uniform conditions (Case 1-3).

\subsection{V-shaped flame front}

V-shaped flame fronts observed in Case 4-9 will be focused on here in order to address relationships between them and incoming mixtures. Since the leading inert shock is oblique, a convex shape obtained here does not necessarily mean that induction length $l_{\text {ind }}$ (distance between a shock front and a flame front) reaches the minimum at the location of its apex. However, the $\mathrm{V}$-shape indicates that relatively reactive mixture comes into its apex. Then, we calculated $l_{\text {ind }}$ at each point on the deflagration front on the assumption that flow behind the leading inert shock is parallel to the surface. This assumption is justified by investigation of flow vectors in that region as mentioned. Location of the shock front was defined as the position where pressure gradient reaches the maximum, and that of the deflagration front as where water mass fraction gradient reaches the maximum. Distribution of $\Phi_{\text {local,in }}$ was also calculated as local equivalence ratio of incoming unburned gas to investigate an effect of reactivity on the flame shape. The results for Case 4 and Case 7 were displayed in Fig. 7 as symbol-line plots. Longitudinal position of the flame front was also plotted as $x_{\text {flame. }}$. We first should note that induction length decreases monotonically outwards from the surface in both cases. This tendency is also retained in the region where $\Phi_{\text {local, in }}$ is below unity. This can be explained by difference of curvature and strength of the incident shock caused by incoming Mach number distribution. Differently from a straight incident shock in uniformly premixed conditions (which can be seen in Fig. 4(a)), post-shock properties (temperature, pressure, etc.) are not uniform along the incident shock. This can be verified by Fig. 8 which shows distributions of post-shock temperatures $T_{p o s t}$ in the two cases considered here. Since $T_{\text {post}}$, which dominantly controls induction length, increases rapidly in both cases, mixture passing across the upper part of the incident shock more readily ignite. This thermal non-uniformity exceeded the effect of non-uniform composition, resulting in the monotonic decrease of $l_{\text {ind }}$ even in learner mixtures.

We will next consider the relationship between location of the V-shaped flame front tip and incoming local equivalence ratio. As shown clearly in Fig. 5, the V-shaped flame front moves little in different non-uniformities. Especially, Case 7-9 experience almost no moving. Further investigation revealed that incoming reactant mixture to the tip has almost the same thermal and chemical properties across different non-uniformities. This is also indicated in Fig. 7 and Fig. 8; $\Phi_{\text {local in }}$ and $T_{\text {post }}$ in the two cases shown at the vertical location of the tip are close to each other. Furthermore, Case 7-9 all encounter the same value of $\Phi_{\text {local, } \text {, }}=1.76$, with $T_{\text {post }}$ limited in the range $1170 \sim 1190 \mathrm{~K}$. These unchanged properties of incoming mixture can be regarded as the cause of fixed position of the flame front tip. This unchanged location and incoming properties of the $\mathrm{V}$-shape tip can also be explained from the shape of the incident shock: As non-uniformity increases, curvature of the incident shock becomes insensitive to its change as indicated in Fig. 5. Therefore, in extremely strong concentration gradients experienced in Case 7-9, streamline with a value of incoming local equivalence ratio comes into similar location on the incident shock, which can consequently generate deflagration at similar locations.

\subsection{Combustion gas properties at the outlet boundary}

Finally, composition and temperature were analyzed at the outlet boundary (the right line of the extended computational domain) to evaluate completeness of combustion there. Distributions of reactant gas compositions and flame temperature computed for Case 4 and Case 7 were plotted as symbol-lines in Fig. 9. In these figures, compositions were shown as mole fractions of hydrogen and oxygen.

In both cases, a large amount of rich reactant gases (hydrogen in the region close to the surface and oxygen away from it) remains unreacted. Mass diffusion effects were not so strong to consume them through diffusive combustion.

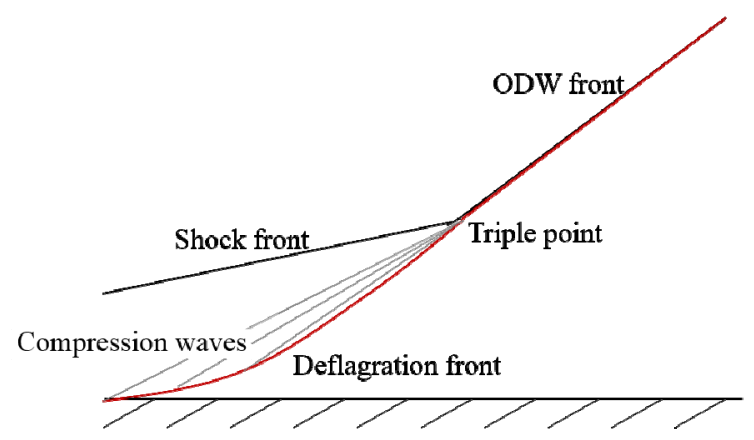

(a) Typical oblique detonation in a uniform mixture

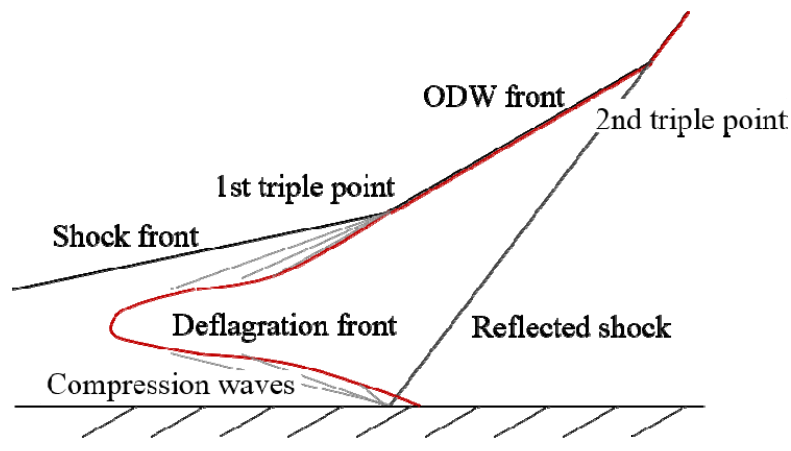

(b) Newly observed oblique detonation in the non-uniform mixtures

Fig. 6. Schematics of the structures of oblique detonaiton waves (ODW) observed. 


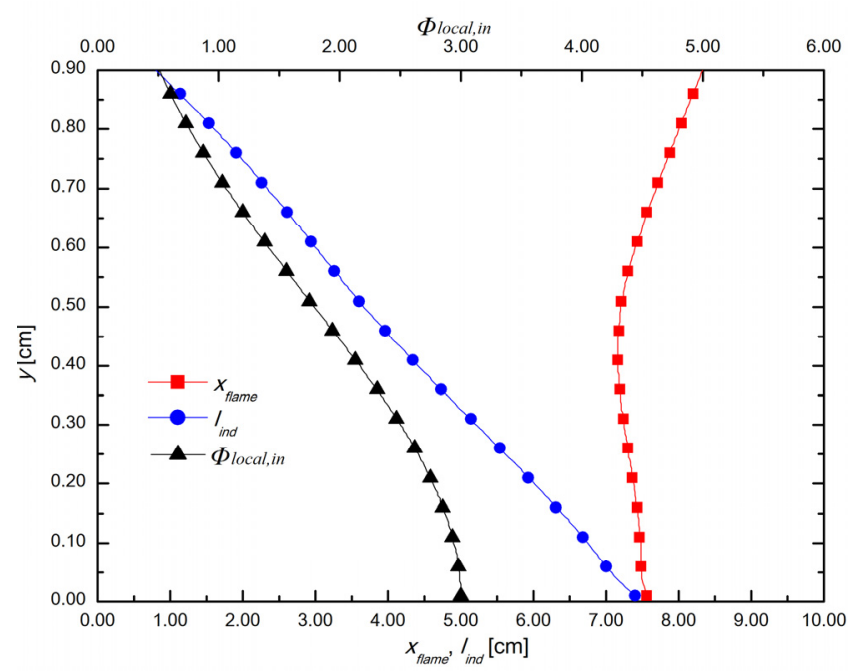

(a) Case $4, \Phi_{\max }=3.00$

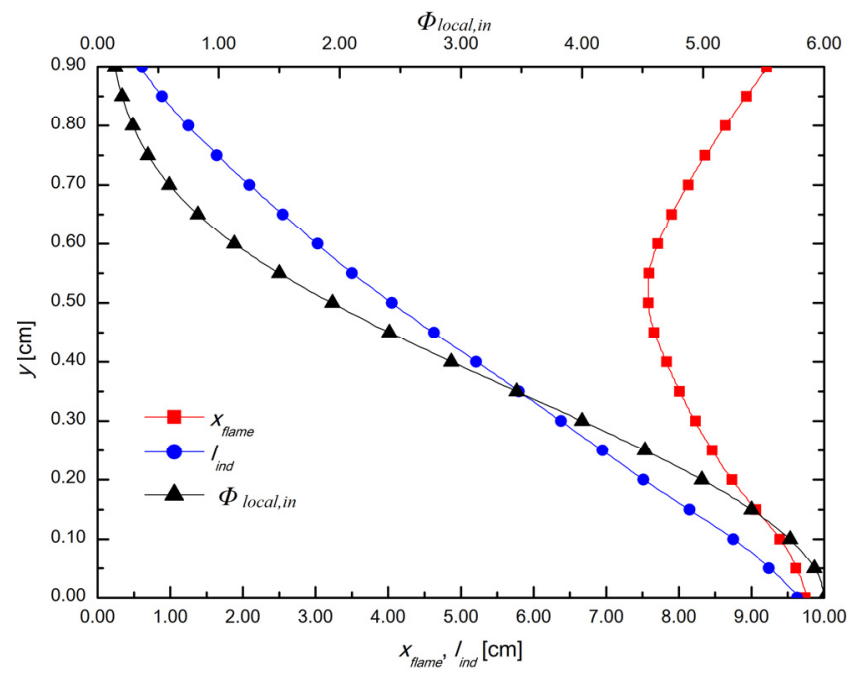

(b) Case 7, $\Phi_{\max }=6.00$

Fig. 7. Relationships between locations of V-shaped flame fronts and incoming local equivalence ratio.

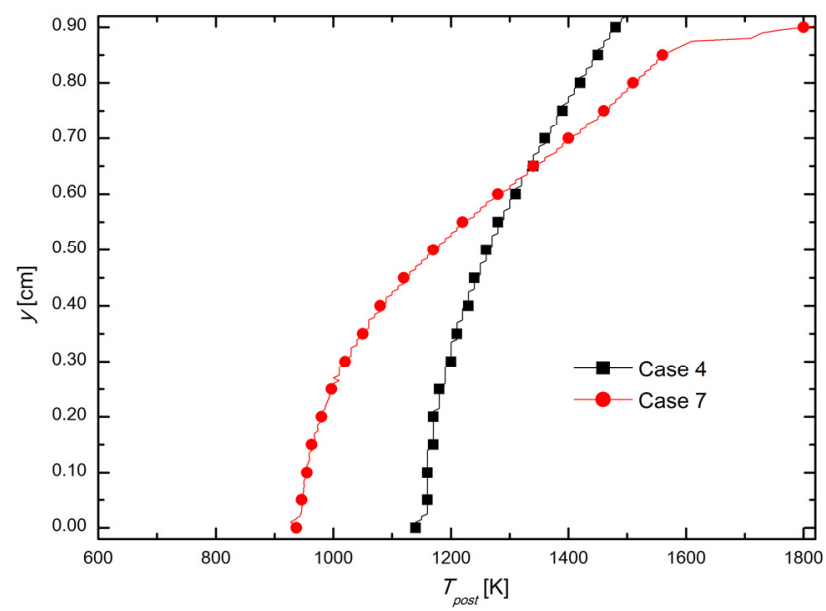

Fig. 8. Distribution of post-shock temperature in Case 4 and Case 7.

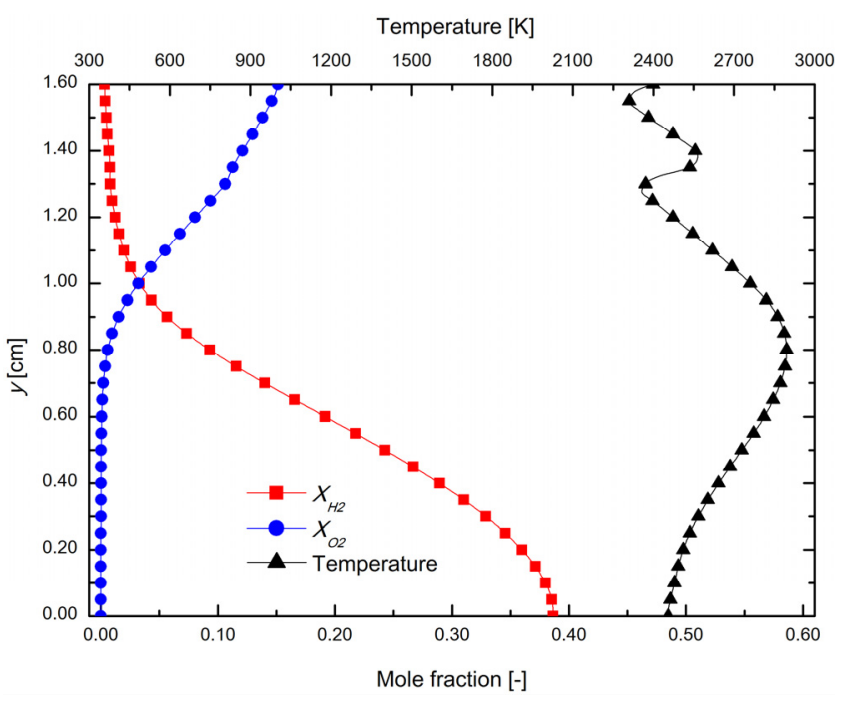

(a) Case 4, $\Phi_{\max }=3.00$

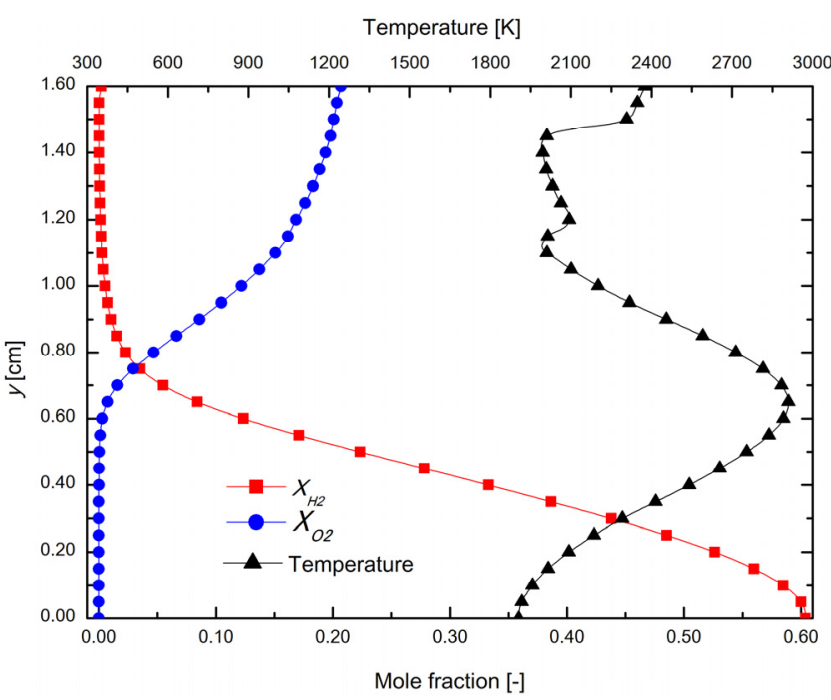

(b) Case 7, $\Phi_{\max }=6.00$

Fig. 9. Distributions of reactants and temperature at the outlet boundary.

However, leaner reactant gases (oxygen in the region close to the surface and hydrogen away from it) are almost completely consumed entirely behind the deflagration front or the detonation front.

Flame temperature at the outlet boundary also has the same qualitative tendency in both cases. High temperature occurs downstream of the V-shaped flame fronts. Since Case 7 encounters the narrower $\mathrm{V}$-shape, the narrower region with high temperature resulted. However, it should be noted that highest temperature is always close to $3000 \mathrm{~K}$, also in stronger non-uniformities of Case 8 and Case 9. These high temperatures behind the deflagration front are combined effects of relatively more reactive composition at its apex $\left(\Phi_{\text {local, } i n}=1.76-2.00\right.$ as previously mentioned $)$ and an additional compression by the reflected shock behind it.

In the outer region $y>1.10 \mathrm{~cm}$, outlet temperature experiences sudden increase twice, which is caused by increase of the shock or detonation angle through the first and second triple point. Thus, decrease of temperature outwards 
can be avoided by existence of the triple points, which enables combustion in a fuel-lean region to be maintained intensive.

\section{Conclusion}

Two-dimensional numerical simulations on a straight wedge stabilized oblique detonation in non-uniformly premixed mixtures were performed solving laminar Navier-Stokes equations including a hydrogen-air detailed reaction mechanism of 9 species and 27 elementary reactions.

The numerical results revealed some newly observed physical features of oblique detonation and shock-induced combustion under non-uniform conditions:

[1] Incomplete premixing brought about the change of a transition pattern from the smooth one to the abrupt one when $\Phi_{\max }=2.00$.

[2] The deflagration front tip deformed into a V-shape around $\Phi_{\max }=3.00$, which caused the triple points to be doubled. The apex of the V-shape did not almost recede under strong non-uniformities.

[3] The first and second triple point in some cases caused transition twice along the incident shock (or detonation) front, while in others no transition occurred.

[4] Second triple point was caused by the intersection of the incident shock (or the detonation front) and a reflected shock from the surface which is the result of accumulation of compression waves emanating from the lower side of the V-shaped flame front.

[5] Induction length decreases monotonically outward resulting from a combined effect of decreasing local equivalence ratio and increasing post-shock temperature behind the incident shock.

[6] Concentration gradients keep a large amount of rich reactant unburned, but lean reactant is always completely consumed regardless of non-uniformity.

Observed features such as a V-shaped flame front and second triple point promise flame stabilization at a certain longitudinal position and intensive combustion far from the surface of the wedge.

Future works will be needed to extend a computational region to a combustor scale and evaluate influence of incomplete premixing on the performance of ODWE, including combustion efficiency and potential thrust.

\section{References}

1) William, H. H., David, T. P., Daniel H. D. and Unmeel, B. M.: Hypersonic Airbreathing Propulsion, AIAA Education Series, Washington, 1994, pp.1-27.

2) Hiraiwa, T., Ito, K., Sato, S., Ueda, S., Tani, K., Tomioka, S. and Kanda, T.: Recent progress in scramjet/combined cycle engines at JAXA, Kakuda space center, Acta Astro., 63 (2008), pp. 565-574.

3) Kanda, T., Tani, K. and Kudo, K.: Conceptual Study of Rocket-Ramjet Combined-Cycle Engine for an AeroSpace Plane, $J$. Prop. Pow., 23, 2 (2007), pp. 301-309.

4) Segal, C.: Scramjet Engine Process and Characteristics, Cambridge, London, 2009, pp. 1-33.

5) Wolanski, P.: Detonative Propulsion, Proc. Combust. Inst., 34 (2013), pp. 125-128.
6) Dunlap, R., Brehm, R. L. and Nicholls, J. A.: A Preliminary Study of the Application of Steady-State Detonative Combustion to a Reaction Engine, Jet. Prop., 28, 7 (1958), pp. 451-456.

7) Wei, H., Hui, Q., ShiBin, L. and ZhenGuo, W.: Research status of key techniques for shock-induced combustion ramjet (shcramjet) engine, Sci. Chi. Tech. Sci., 53, 1 (2010), pp. 220-226.

8) Lee, J. H. S.: The Detonation Phenomenon, Cambridge University Press, 2008, pp. 1-25.

9) Shepherd, J. E.: Detonation in gases, Proc. Combust. Inst., 32 (2009), pp. 83-98.

10) Sislan, J. P., Dudebout, R., Schumacher, J., Islam, M. and Redford, T.: Incomplete Mixing and Off-Design Effects on Shock-Induced Combustion Ramjet Performance, J. Prop. Pow., 16, 1 (2000), pp. 41-48.

11) Wei, H., Shi-bin, L. Y. and Zhen-guo, W.: Performance evaluation and parametric analysis on cantilevered ramp injector in supersonic flows, Acta Astro., 84 (2013), pp. 141-152.

12) Yen-W, W. and Sislan, J. P.: Numerical Simulation of Gaseous Hydrocarbon Fuel Injjection in a Hypersonic Inlet, J. Prop. Pow., 26, 5 (2010), pp. 1114-1124.

13) Verreault, J., Higgins, A. J. and Stowe, R. A.: Formation and Structure of Steady Oblique and Conical Detonation Waves, AIAA J., 50, 8 (2012), pp. 1766-1772.

14) Maeda, S., Kasahara, J. and Matsuo, A.: Oblique detonation wave stability around a spherical projectile by a high time resolution optical observation, Combust. Flame., 159 (2012), pp. 887-896.

15) Ess, P. R., Allen, C. B.: Blunt-Body Generated Detonation in Viscous Hypersonic Ducted Flows, J. Prop. Pow., 21, 4 (2005), pp. 667-680.

16) Kamel, M. R., Morris, C. I., Stouklov, I. G. and Hanson, R. K.: PLIF imaging of hypersonic reactive flow around blunt bodies, Symp. Combust., 26, 2 (1996), pp. 2909-2915.

17) Cambier, J. L., Adelman, H. and Menees, G. P.: Numerical Simulation of an Oblique Detonation Wave Engine, Jet. Prop., 6, 3 (1990), pp. 315-323.

18) Vlasenko, V. V. and Sabel'nikov, V. A.: Numerical Simulation of Inviscid Flows with Hydrogen Combustion Behind Shock Waves and in Detonation Waves, Combust. Explo. Shock Waves, 31, 3 (1995), pp. 376-389.

19) Silva, L. F. F. and Deshaies, B.: Stabilization of an Oblique Detonation Wave by a Wedge: A Parametric Numerical Study, Combust. Flame. 121 (2000), pp. 152-166.

20) Konnov, A. A.: Development and validation of a detailed reaction mechanism for the combustion modeling, Eurasian Chem. Tech. J., 2 (2000), pp. 257-264.

21) Yee, H. C.: Upwind and Symmetric Shock-Capturing Schemes, NASA TM-1987-89464, 1987.

22) Jameson, A. and Yoon, S.: Lower-Upper Implicit Schemes with Multiple Grids for the Euler Equations, AIAA J., 25, 7 (1987), pp. 929-935.

23) Ju, Y.: Lower-Upper Scheme for Chemically Reacting Flow with Finite Rate Chemistry, AIAA J., 33, 8 (1995), pp. 1418-1425.

24) Gross, R. A., and Chinitz, W.: A Study of Supersonic Combustion, J. Aero. Sci., 27, 7 (1960), pp. 517-524.

25) Menees, G. P., Adelman, H. G. and Cambier, J. L.: Analytical and Experimental Investigations of the Oblique Detonation Wave Engine Concept, NASA TM-1991-102839, 1991.

26) Choi, J. Y., Kim, D. W., Jeung, I. S., Ma, F. and Yang, V.: Cell like structure of unstable oblique detonation wavve from high^${ }^{\wedge}$ resolution numerical simulation, Proc. Combust. Inst., 31 (2007), pp. 2473-2480. 